\title{
INVESTIGATION OF AIRCRAFT WAKE VORTICES WITH PHASED MICROPHONE ARRAYS
}

\author{
Ulf Michel ${ }^{*}$ and Peer Böhning ${ }^{\dagger}$ \\ German Aerospace Center (DLR), Institute of Propulsion Technology \\ Müller-Breslau-Str. 8, 10623 Berlin, Germany
}

\begin{abstract}
Wake vortices of landing aircraft emit a faint noise that is audible when wind speed is low and might be related to aerodynamic parameters of the vortices. Results from a test of the capability of phased microphone arrays for the investigation of wake vortices are presented. It is shown that it is possible to estimate the frequency spectrum of wake-vortex noise, its spatial sound source distribution and even the trajectory of the two counter-rotating vortices. The paper presents vortex-noise frequency spectra and sound source distributions of the wake vortices measured behind a Boeing 737, Boeing 757 and an Airbus A320. The phased microphone array technique is shown to be an appropriate tool for wake vortex investigations.
\end{abstract}

\section{Introduction}

The wake vortices of aircraft are a research subject of continued interest, driven by the potential hazards to aircraft encountering the ensuing wake turbulence. The danger may even rise in the future due to increasing size of aircraft, and decreasing lateral and vertical separations that are necessary to cope for the air traffic needs en-route and in the terminal areas.

While the origin of the wake vortices is well recognized, the knowledge of the development of the flow field in the vortices, their final breakup and possible control is still limited. This situation prompted the creation of three European research projects, (i) $C$-WAKE devoted to wake vortex characterization and control, (ii) I-WAKE for wake vortex instrumentation and detection, and (iii) $S-W A K E$ for wake vortex safety assessment.

Experience shows that the wake vortices of aircraft are audible. Based on this observation we proposed to start an attempt to characterize and possibly even localize wake vortices by means of their emitted noise. The use of ordinary microphones without postprocessing of signals would likely be inappropriate because these signals would suffer under severe influence of background noise from the aircraft itself, from wind, and from other noise sources on the ground. However, the technique of the phased microphone array might be an appropriate tool. The beamforming algorithm will suppress background noise from other directions than the vortices and might even allow a localization and tracking of the sound sources.

The capability of this technique for wake-vortex research is currently investigated by the German Aerospace Research Center $(D L R)$ within the above mentioned Euro-

\footnotetext{
*ulf.michel@dlr.de, phone : +49 30 310006-26, fax : -39

†peer.boehning@dlr.de, phone : +49 30 310006-48, fax : -39

Copyright (@) 2002 by Ulf Michel and Peer Böhning. Published by the American Institute of Aeronautics and
}

pean research project $C$-WAKE. Since the microphone array technique has never been applied to wake vortex noise before, a proof of the technique's capability to capture wake vortex noise was first attempted. For this purpose, a large phased array of microphones was erected within the fence of Berlin's Airport Schönefeld. The noise of landing commercial aircraft was recorded over a duration long enough to also include wake-vortex noise emission. This paper presents some results obtained in this measurement campaign.

The sources of wake-vortex noise are describabed by the quadrupole source term of the Lighthill theory, see, e.g., Michalke and Michel. ${ }^{1}$ The source strength is proportional to the second time derivative of the square of the component of the velocity fluctuations toward the observer position. The radiation effectiveness is described by the coherence and interference between neighboring source positions and generally increases with the coherence length scale of the source term within the source region. The physical origin of the turbulent velocity fluctuations inside the wake vortices is not known. However, it is known that wake vortices are subject to various instability mechanisms that lead to their final breakup. It is also known that vortices with an axial velocity component inside the core are subject to an absolute instability as shown by Michalke. ${ }^{2}$

\section{Phased microphone array technique}

As a result of advances in computer technology and the availability of relatively cheap microphones, the localization of sound sources on fixed and moving objects with phased microphone arrays is slowly becoming a standard method. It was first proposed for aeroacoustic applications by Billingsley \& Kinns. ${ }^{3}$ A recent application to moving sources on a flying Airbus A340 is reported by Piet et al. ${ }^{4}$ where a detailed description of the beamforming technique on moving objects is included. It is shown by Siller et 


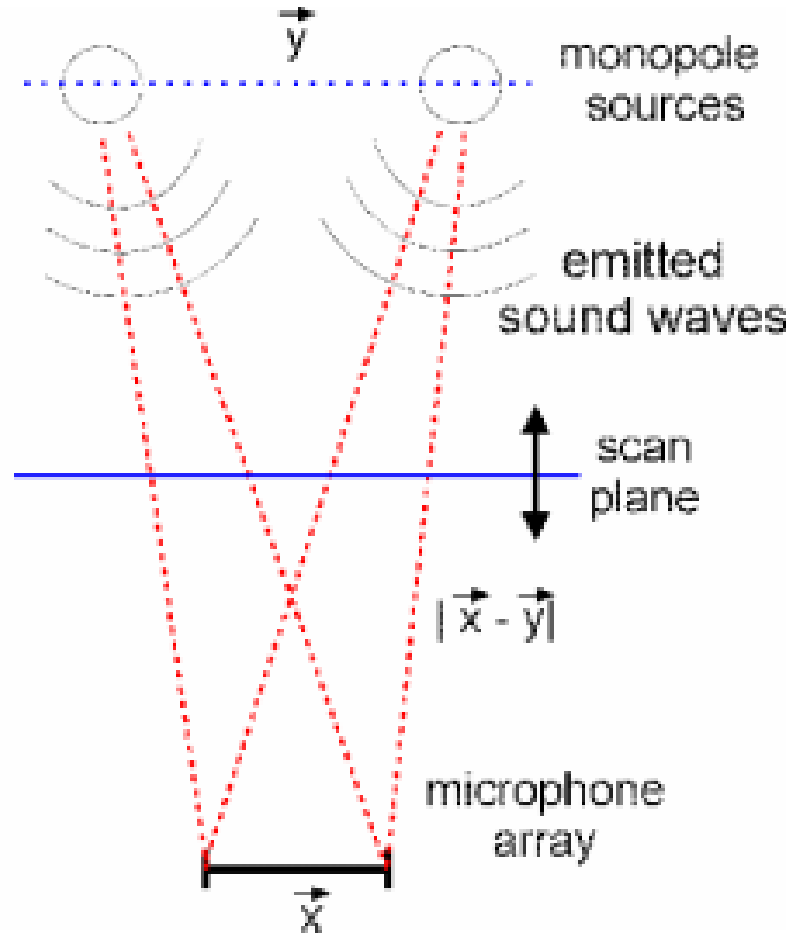

Fig. 1 Principle of the microphone array technique

al. ${ }^{5}$ for an aero-engine on a static free-field testbed, that the source localized on the nozzle can be correlated with a pressure signal from inside the combustion chamber to investigate the contribution of combustion noise to the noise emission of this engine. The technique can also be applied to the investigation of engine noise in flight. The buzz-saw noise of the two engines of an Airbus A320 is compared by Siller \& Michel $^{6}$ by focusing to each engine inlet during take-off.

The application to wake-vortex noise is illustrated in Fig. 1 where the situation is shown in a vertical plane crossing the axes of the two counter-rotating wake vortices, which are assumed as line sources. The sound waves emitted from any position on the two line sources will arrive at the microphones of the array after a time delay determined by the various propagation distances and the speed of sound. The differences in the time delays are the basis of the phased array analysis.

The data reduction is based on the assumption that the sound waves are sperical and that their amplitudes are only a function of distance. This is strictly true only for monopole sources. However, a close inspection of the phases of dipole and quadrupole sources reveals that the phases are almost constant on spheres in the acoustic far field of these sources, while the amplitudes are functions of emission angle. Therefore, the beamforming algorithm may also be applied to dipole and quadrupole sources as long as the aperture of the array (ratio of array size over distance between the sources and the array) is not too large. The beamforming results in an averaging of the directivity over the surface of the array.

During the data reduction, the microphone array is focused to many source positions, assumed to be located in a scan plane as indicated in Fig. 1. Ideally, the assumed scan plane would include the two vortex lines which are slightly inclined in the flight direction because the vortex altitude becomes smaller as a function of time. Focussing involves a modification of amplitude and phase of each microphone signal to compensate for the effect of different propagation distances between the assumed focal position and each microphone, see Piet at al. ${ }^{4}$ for details.

Averaging of all focused microphone signals suppresses noise originating away from the focus position while noise originating from the focus position is preserved. The suppression capability is higher in the horizontal direction than in the vertical direction. Focusing the array allows the frequency spectrum of the noise emitted by a sound source of known position to be estimated even in the presence of other sources or background noise. The level of permissable background noise depends on the beam pattern of the array which is a function of layout and number of microphones. Assuming all sound sources are located in the scan plane, a map-like distribution of sound sources is obtained by focusing at points of a grid in this scan plane. It is shown by Piet et al. ${ }^{4}$ that these maps must be computed for frequency bands because the resolution of the array is frequency dependent. Also, the map level is not necessarily a measure of the source level.

The altitude of the scan plane has to be estimated. If it is assumed lower than the actual vortex lines, as shown in Fig. 1, the apparent distance of the vortices will be smaller. On the other hand the apparent distance will be larger if the scan plane is located in a higher altitude than the vortices.

If the apparent sound source distribution is calculated for a sequence of short time intervals, the wake vortex evolution in time can be studied. In the results presented in this paper, the altitude of the scan plane was kept constant. However, it should consider the sink velocity or a tracking algorithm which is proposed in this paper.

\section{Experimental setup}

The test campaign was conducted inside the fence of Berlin's Schönefeld-Airport in May 2000 on commercial aircraft on their final landing approach to runway 25L. The array was constructed east of the localizer protection zone about $700 \mathrm{~m}$ before the runway threshold. The flyover altitudes were between $40 \mathrm{~m}$ and $60 \mathrm{~m}$, the ground speeds about $v=70 \mathrm{~m} / \mathrm{s}$.

The microphone array was layed out in circles with exponentially increasing radius. Eight microphones were uni- 
formely distributed on each circle. The diameter of the outer circle was $12.5 \mathrm{~m}$. The ratio between two adjacent radii was 0.794 which is equal to the frequency ratio of two adjacent one-third octave bands. The objective of this choice was to enable a constant beamwidth for all one-third octave bands for the noise source localization on the aircraft. (See Piet et al. ${ }^{4}$ for a dicussion of beam patterns.) A large size subarray of 64 microphones with an outer diamter of $12.5 \mathrm{~m}$ was used for the wake-vortex study.

The microphones were mounted with grazing incidence on moisture resistant wooden plates. The wooden structure consisted of a square of $6 \mathrm{~m}$ by $6 \mathrm{~m}$ with 8 radial extensions $2 \mathrm{~m}$ wide for every 45 degrees. The plate was carefully contructed with a horizontal orientation to simplify the determination of the microphone positions.

The data were acquired in two stages. The first stage was to record the microphone data of the aircraft fly-over for a later source localization on the aircraft itself. Recording started about $2 \mathrm{~s}$ before the aircraft passed the array and had a duration of $6 \mathrm{~s}$. The second stage was to record the noise of the wake vortices behind the aircraft. Since wake-vortex noise is much quieter than the noise emitted by the aircraft itself, the signal amplification of all channels was increased by $40 \mathrm{~dB}$ within a small fraction of a second. Recording of the second stage started about $4 \mathrm{~s}$ after the aircraft had passed the array and had a duration of $30 \mathrm{~s}$. The microphone signals of both stages, fly-over and wake vortex, were sampled using 16-bit A/D converters with a sampling frequency of $f=33 \mathrm{kHz}$. All data were first stored in the memory of the acquisition computer and then copied to the hard disk.

The aircraft ground speed was determined by two line cameras positioned before and after the array on the flight path with a known distance. The altitude of the fly-over was determined by an array of three laser distance meters separated by $10 \mathrm{~m}$ perpendicular to the flight path. These distance meters scanned for a target with a frequency of 500 $\mathrm{Hz}$ and created a trigger signal when they acquired a target during a fly-over. The analog signals of the line cameras and the trigger pulses of the distance meters were recorded together with the microphone signals.

Data were obtained for aircraft of different types, at flyover altitudes between $40 \mathrm{~m}$ and $60 \mathrm{~m}$, and ground speeds of approximately $v=70 \mathrm{~m} / \mathrm{s}$.

\section{Data processing}

\section{Fly-over data}

The fly-over data were processed in order to verify flight parameters such as aircraft altitude, ground speed, lateral offset of the flight path relative to the runway center line and the exact time when the aircraft crossed the array cen- ter. Lateral deviation is especially important for the interpretation of wake vortex data. The algorithm applied for the processing of the fly-over data is based on the delayand-sum beamformer in the time domain and allows the focus to be fixed on the moving aircraft. The result of the processing is the sound source distribution in the scan plane as shown in Figs. 2, 5, and 8 for the $1000 \mathrm{~Hz}$ one-third octave band.

\section{Wake vortex data}

The wake vortex data were processed by an algorithm for stationary sound sources based on the delay-and-sum beamformer. Since only a slow change of vortex positions is expected, sources are assumed for simplicity to be stationary within a sufficient short time interval. The wake vortex data were split into sequences of short time intervals. For each interval the sound source distribution in the scan plane was processed. The altitude of the scan plane was not adjusted. This method was chosen since the exact trajectory of the vortices was not known. The sequence of the sound source distribution maps provides information on the vortex evolution in time.

The quality of the sound source distribution depends strongly on the size of the time intervals. A long interval corresponds to a long averaging time which improves the signal to noise ratio but increases "smearing" due to source movement. Experience showed an interval size of $0.5 \mathrm{sec}$ to be a good compromise.

The altitude of the scan plane was fixed to $h=40 \mathrm{~m}$ for all intervals. Since the altitude of the wake vortices decreases with time a parallax effect is expected. As a result, a virtual sidewards movement of the vortices is expected to be observed.

A general problem of array techniques is the existence of sidelobes. Sidelobes appear in source maps as "virtual sources" caused by strong sources some distance away from the focus point. These virtual sources may lead to misinterpretations of a source distribution. By means of simulations, it was validated that the sound source distribution interpreted as wake vortex is not a virtual source caused by the much louder landed aircraft. The positions of two simulated moving monopole sources emitting random noise were defined according to dominant sources in the source distribution maps calculated for the aircraft flyovers.

\section{Results and Discussion}

Fig. 2 shows the source maps of the one-third octave band (TOB) $f=315 \mathrm{~Hz}$ of a landing Airbus A320-200 in an altitude of $h=51 \mathrm{~m}$ and with a ground speed of $v=72 \mathrm{~m} / \mathrm{sec}$. The scan plane was assumed at 51 meters above the array with a glide slope of 3 degrees. The color 
scales of all following maps are referenced to their individual maximum levels. The color range is always $10 \mathrm{~dB}$ where black indicates strong and white weak sources. The sound emission of the TOB $f=315 \mathrm{~Hz}$ is seen to be dominated by the aircraft's engines. The aircraft's silhouette was placed arbitrarily on top of the map. According to the experience with other tests it can be assumed that the sound at this frequency is dominated by exhaust noise at engine speeds typical for the landing approach.

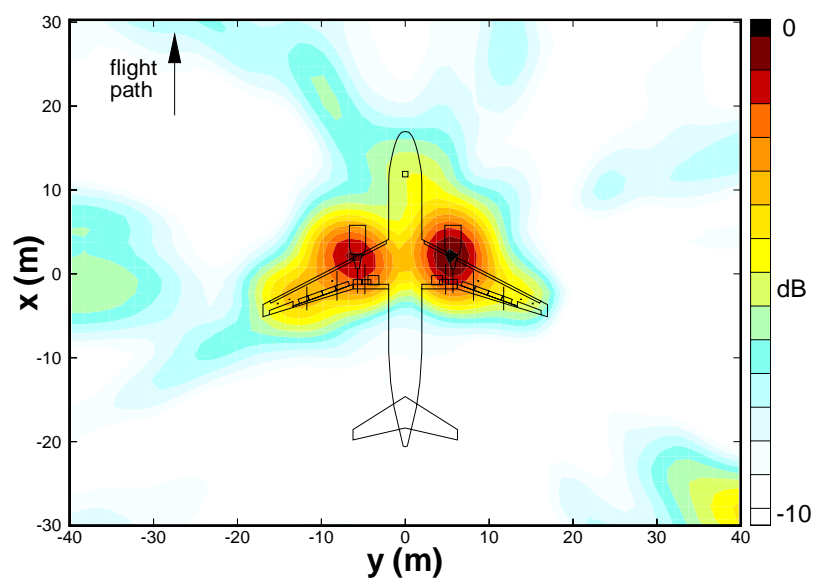

Fig. 2 Source map of an A320-200 during fly-over in the $315 \mathrm{~Hz}$ one-third octave band, processed with moving focus.

The source maps of the TOB $f=315 \mathrm{~Hz}$ about $6 \mathrm{~s}$ and $10 \mathrm{~s}$ behind the aircraft are shown in Figs. 3 and 4. The aircraft's flight path is indicated as in Fig. 2. The two vertical dashed lines indicate the wing span of the aircraft. A horizontal scan plane in an altitude of $40 \mathrm{~m}$ was assumed in both figures. A source distribution along two vertical parallel lines is clearly visible. These sources are beleaved to be attributed to the two counter-rotating wake vortices behind the aircraft. The variation of apparent source intensity was found in all vortex-noise maps and seems to be typcial of wake-vortex noise.

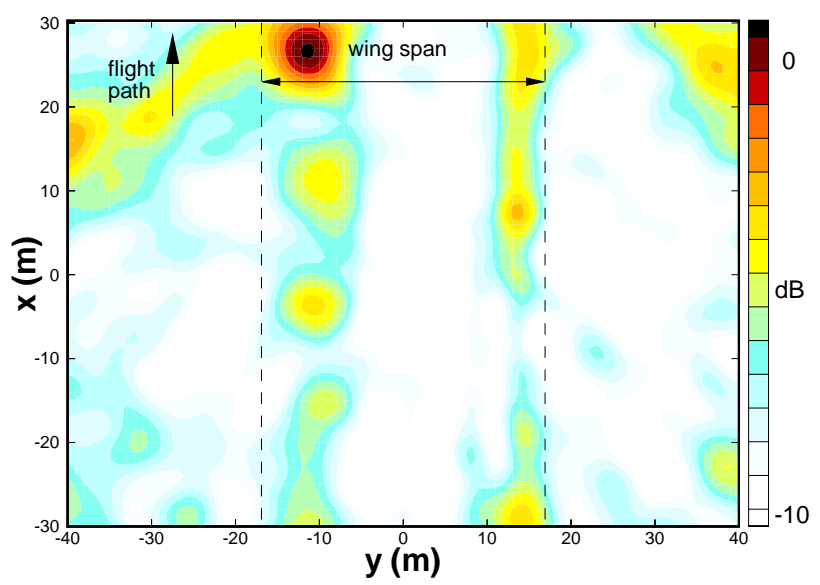

Fig. 3 Source map $6 \mathrm{~s}$ after an A320-200 passed the microphone array, processed with stationary focus

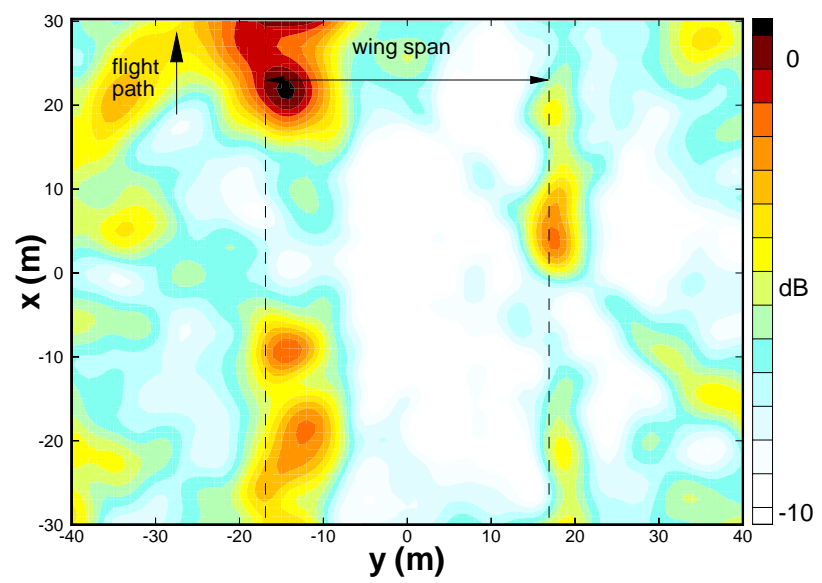

Fig. 4 Source map $10 \mathrm{~s}$ after an A320-200 passed the microphone array, processed with stationary focus

By comparing the source distributions for 2 different times after the pass-by of the aircraft (Figs. 3 and 4), a slight increase of the spacing between the parallel source lines with increasing time becomes evident. This increase is caused by two effects. Firstly, the counter-rotating vortices increase their spacing due to the ground effect (influence of the mirror vortices below the ground). Secondly, the spacing appears to increase due to the parallax effect explained before. As the exact wake vortex sink rate was not known, it is difficult to separate the two effects with the available equipment. 


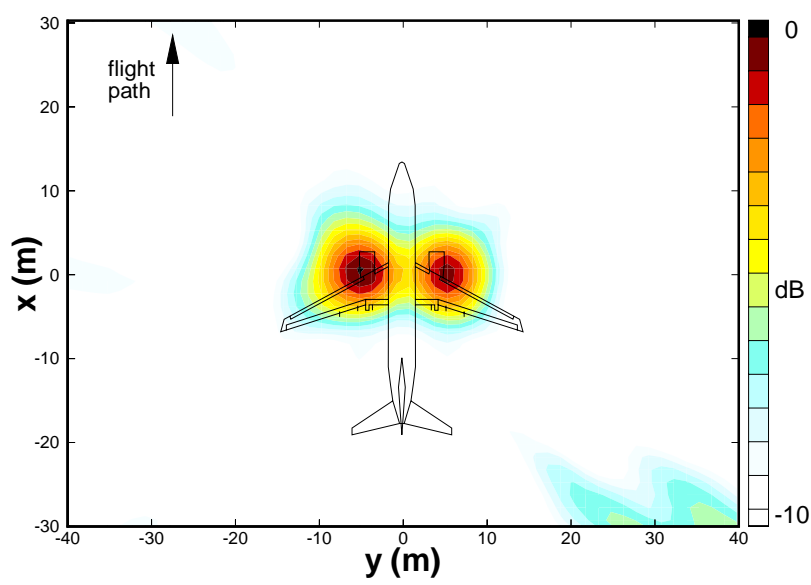

Fig. 5 Source map of a Boeing 737-300 during fly-over in the $315 \mathrm{~Hz}$ one-third octave band.

We now turn to the next aircraft type presented here. Fig. 5 shows the source maps in the TOB $f=315 \mathrm{~Hz}$ of a landing Boeing 737-300 at an altitude of $h=49 \mathrm{~m}$ and a ground speed $v=72 \mathrm{~m} / \mathrm{sec}$. As before in the case of the A $320-200$ the sound emission in the TOB $f=315 \mathrm{~Hz}$ is dominated by the aircraft's engines.

In Figs. 6 and 7 the source map of the TOB $f=315 \mathrm{~Hz}$ about 6 and 10 secionds behind the aircraft are shown. Behind the Boeing 737-300, a source distribution along two parallel lines can also be observed. The movement of the wake vortices in the y-direction is caused by cross-wind.

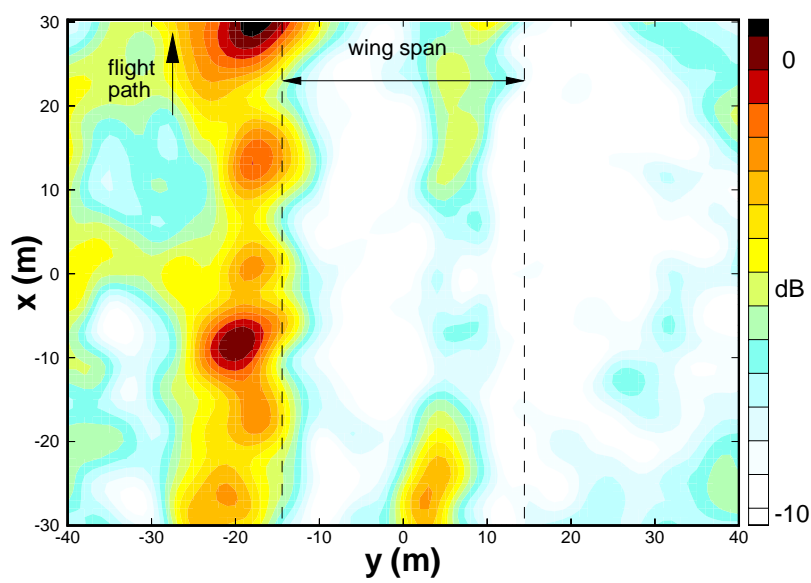

Fig. 6 Source map 6 seconds after a Boeing 737-300 passed the microphone array.

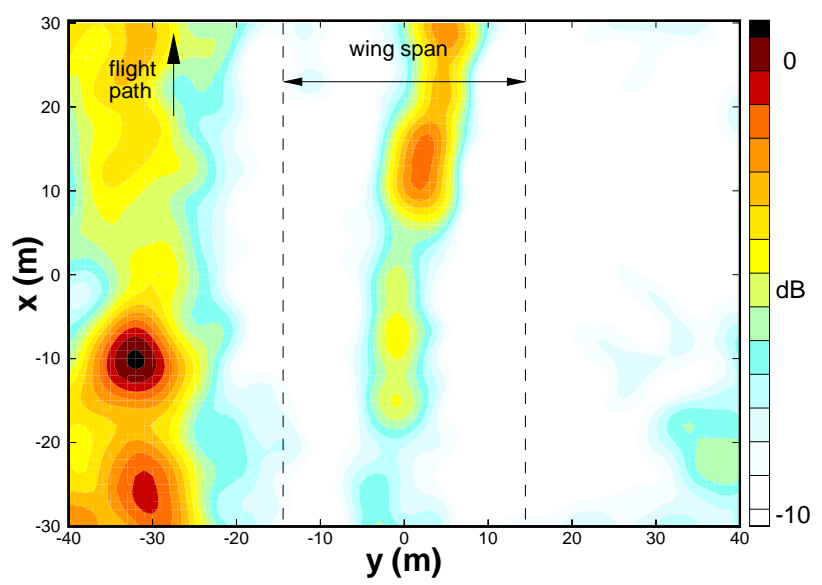

Fig. 7 Source map 10 seconds after a Boeing 737-300 passed the microphone array.

Fig. 8 shows the source map in the TOB $f=315 \mathrm{~Hz}$ of a landing Boeing 757-200 at an altitude of $h=49 \mathrm{~m}$ and an ground speed $v=67 \mathrm{~m} / \mathrm{sec}$. The sound emission of the TOB $f=315 \mathrm{~Hz}$ again is dominated by the engines. The two engines have a very different noise signature, whose cause is very likely combustion noise, which can differ substantially between engines.

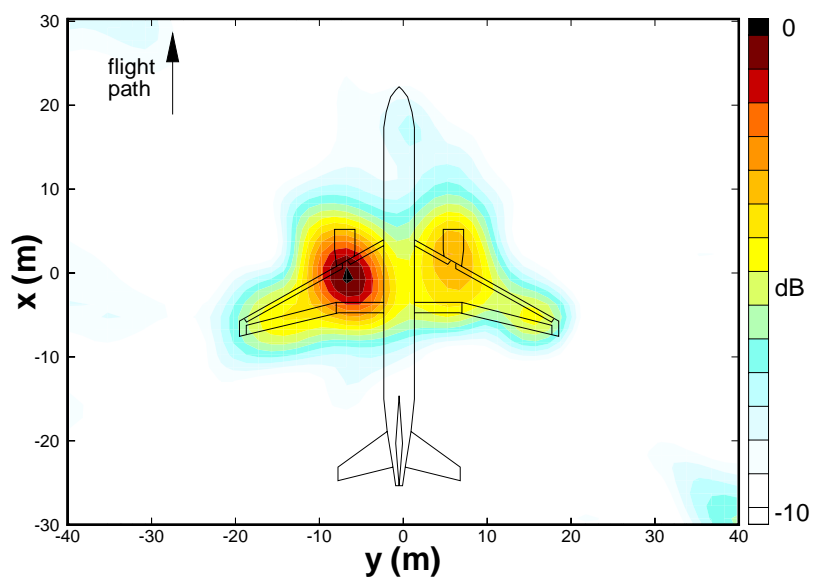

Fig. 8 Source map of an 757-200 during fly-over in the $315 \mathrm{~Hz}$ one-third octave band.

The corresonding source maps 6 and 10 seconds after the aircraft has passed are shown in Figs. 9 and 10, respectively. In comparison to the previous maps of the A320-200 and the Boeing 737-300 it can be noticed that the vortices seem to be much more concentrated. If this can be related to the longevity of the vortices is not known yet, but it is known that the wake vortices of the 757 have an exceptionally long live.

The resolution of the maps is proportional to the size of the array (here with a diameter of 12.5 meters) and inversely proportional to the the distance of the vortices from the array. It can be concluded from the maps that the res- 


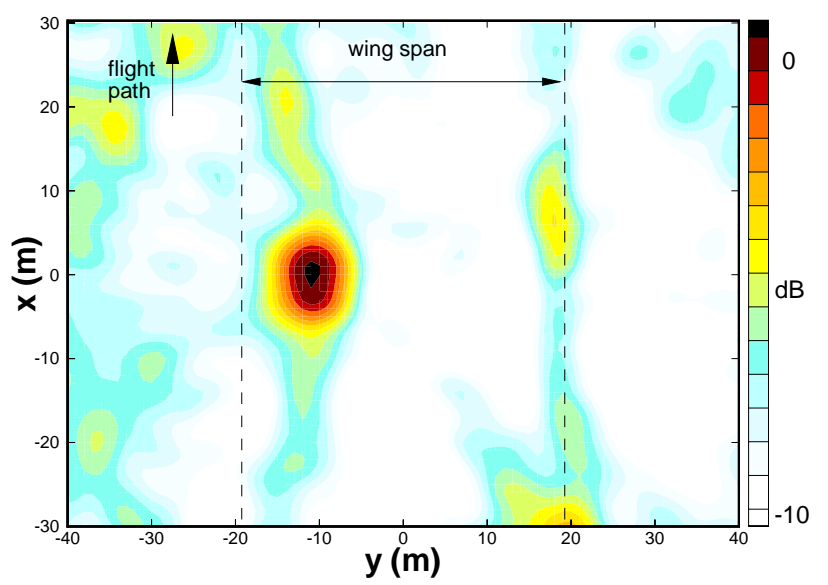

Fig. 9 Source map 6 seconds after a 757-200 passed the microphone array.

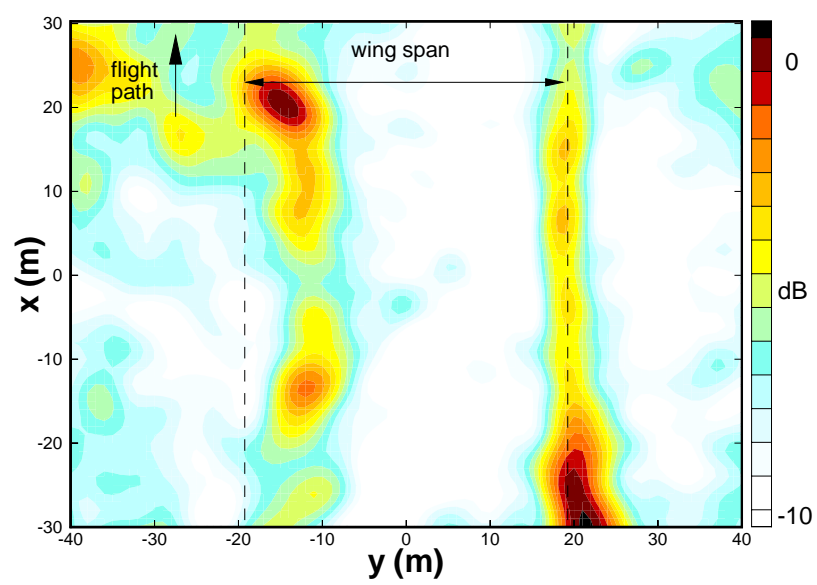

Fig. 10 Source map 10 seconds after a 757-200 passed the microphone array.

olution is better than necessary for a separation of the two vortices. In order to reduce the resolution, the size of the array could be reduced substantially or the altitude of the vortices above the array increased before the two vortex lines would merge on the maps.

Figs. 11 to 13 show the averaged power spectral densities of the three different aircraft types. The average was taken from the focused spectra of all grid points along the two visible source lines in the corresponding maps. All spectra are strongly dominated by low-frequency noise. However, part of the decay with increasing frequency is due to the focusing procedure.

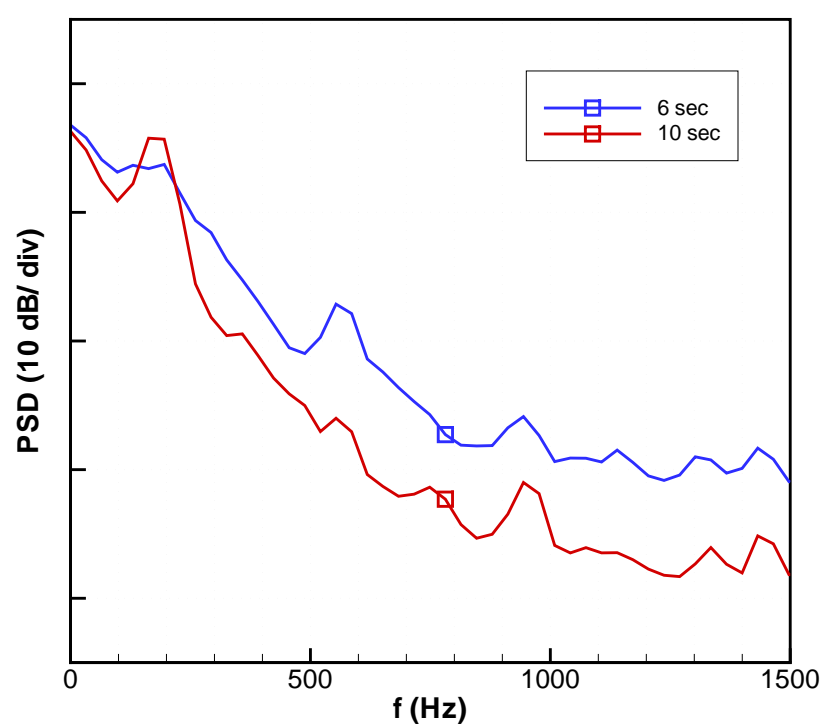

Fig. 11 Spectra of the wake vortex noise behind an A320-200.

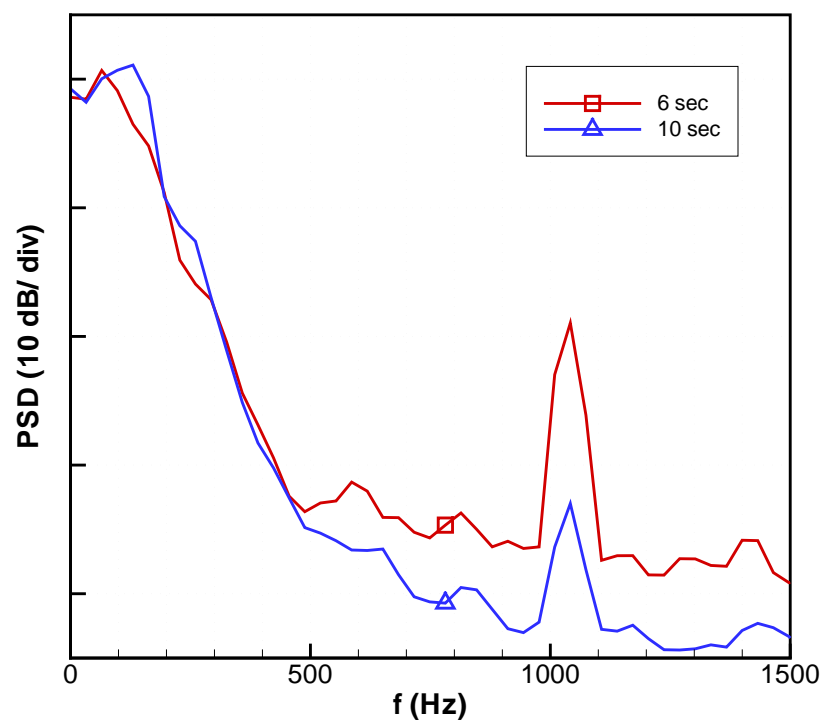

Fig. 12 Spectra of the wake vortex noise behind a Boeing 737-300.

The tonal component visible in all spectra at around $1 \mathrm{kHz}$ is a background noise or spurious noise because it cannot be localized with the array in the scan plane. It features a decreasing level with time, which my indicate that it is related to noise emitted by the aircraft itself, which has reached distances of about 400 meters and 700 meters from the array after 6 and 10 seconds, respectively. (Touchdown is at about 800 meters.) This shows that the capability of an array to reduce background noise is limited for a phased array with only 64 microphones.

The spectral levels are normalized to a distance of 1 meter. The level of the top grid line in all plots is the same. By comparing the spectra for the three aircraft types we note 


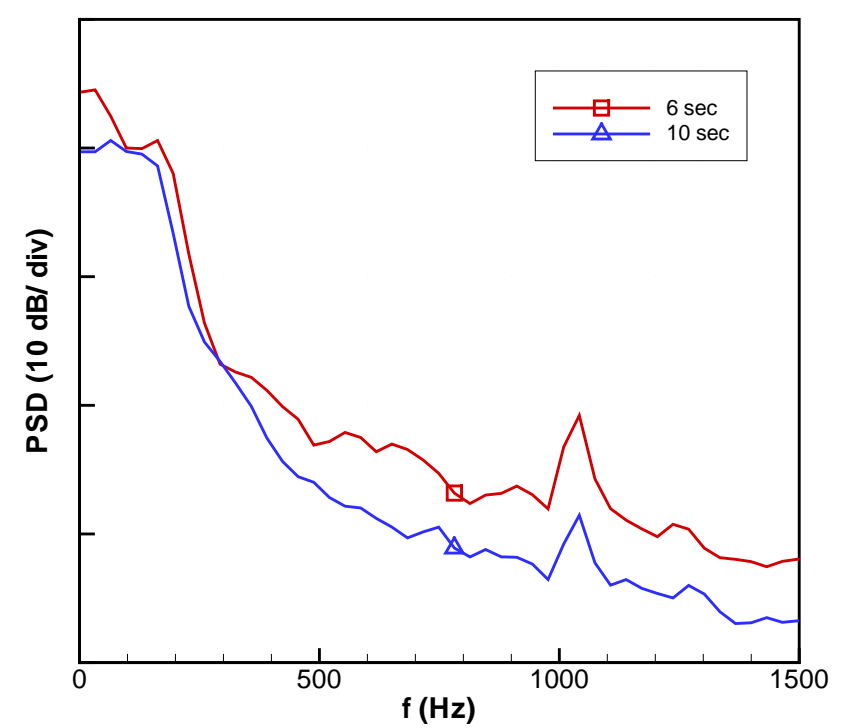

Fig. 13 Spectra of the wake vortex noise behind a 757-200.

that the 737-300 has the highest decay of more than $30 \mathrm{~dB}$ between the peak at about $100 \mathrm{~Hz}$ and $500 \mathrm{~Hz}$. The spectral peak level of the 757-200 is slightly higher and the peak slightly wider and the decay rate is smaller in comparison to the 737. The peak level of the A320-200 is about $5 \mathrm{~dB}$ smaller, the peak frequency is about $200 \mathrm{~Hz}$ and the decay rate is rather small in comparison to the other types.

The nominal frequency of the one-third octave band of $315 \mathrm{~Hz}$ of all maps in this report is a compromise between localization capability and resolution. As one can see from the spectra, a localisation at lower frequencies would yield higher map levels, however, the resolution of the maps would be lost because the resolution of the array is proportional to the wavelength of the sound.

\section{Conclusions}

The prime objective of this study was a demonstration of the capabilities of vortex-noise measurements with phased microphone arrays. The results prove that this technique is an appropriate tool for the estimation of the frequency spectra of wake-vortex noise and even for the localization of wake vortices. Wake-vortex noise is weak and may easily be obscured by background noise or wind noise. Therefore, a large number of microphones is needed to ensure a high amplification of the microphone array and low sidelobes.

A further objective of the test was to define the requirements for a dedicated vortex noise measuring equipment.

- The array has to be prefabricated to allow easy relocation of the test site and to lower the costs of erecting an array with a large number of microphones.

- The number of microphones must be high to ensure a high amplification of the microphone array with low sidelobes. 128 microphones seem to be a minimum.

- An array size of 5 meters might be sufficient up to vortex altitudes of 100 meters for a localization at 315 Hz. The vortex altitude could be even as high as 200 meters if the analysis could be performed at $630 \mathrm{~Hz}$.

- The equipment should include the capability to estimate the altitude of the vortices. This would require a second array of microphones positioned laterally of the first array. The vortex positions can be localized by triangulation.

A further experience gained in the test was that the altitude of the wake vortices was too low, because the ground effects and the influences of turbulence on the vortex development were already large. New tests will be performed in typical altitudes above 100 meters.

Further measurements need to be conducted in order to check out the limits of the microphone array technique for wake-vortex investigation with respect to vortex altitude and life time as well as meteorological conditions such as wind speed.

An important objective of future measurements will be a correlation of the acoustic data with the velocity profiles within the vortices as measured by LIDAR simultaneously. This correlation may allow the development of a description of the noise characteristics of a wake vortex as a function of flow data.

\section{$\underline{\text { Acknowledgments }}$}

These tests were supported by the European Commission through the C-WAKE (Wake Vortex Characterization and Control) Project. The authors appreciate the important support of the Airport Schönefeld of Berlin and the Deutsche Flugsicherung (German Air Navigation Services), which allowed the installation and operation of the equipment close to the runway end. Especial thanks go to Mrs. Baierova of the environmental office of the airport for her support and interest in this study.

\section{References}

\footnotetext{
${ }^{1}$ Michalke, A. and Michel, U., "Prediction of Jet-Noise in Flight from Static Tests," J. Sound Vib., Vol. 67, 1979, pp. 341-367.

${ }^{2}$ Michalke, A., "Absolute inviscid instability of a ring jet with backflow and swirl," European Journal of Mechanics, B/Fluids, Vol. 18, 1999, pp. 3-12.

${ }^{3}$ Billingsley, J. and Kinns, R., "The Acoustic Telescope," J. Sound Vib., Vol. 48, 1976, pp. 485-510.

${ }^{4}$ Piet, J., Michel, U., and Böhning, P., "Localization of the Acoustic Sources of the A340 with a large Phased Microphone Array during Flight Tests," AIAA/CEAS Paper 2002-2506, 2002.
} 
${ }^{5}$ Siller, H., Arnold, F., and Michel, U., "Investigation of Aero-Engine Core-noise Using a Phased Microphone Array," AIAA/CEAS Paper 20012269, 2001.

${ }^{6}$ Siller, H. and Michel, U., "Buzz-Saw Noise Spectra and Directivity from Flyover Tests," AIAA Paper 2002-2562, 2002, 8th AIAA/CEAS Aeroacoustics Conference, Brekenridge, Co, June 17-19, 2002. 